Supporting Information

\title{
Precisely Tunable Humidity Color Indicator Based on Photonic Polymer Films
}

Seo-Hyun Jung, ${ }^{a, b}$, Hyun Tae Lee ${ }^{a, d}$, Min Ji Park ${ }^{a, d}$, Bogyu Lim ${ }^{a}$,Byong Chon Park, Yu Jin Jung ${ }^{a}$, Hoyoul Kong ${ }^{a}$, Do-Hoon Hwang ${ }^{d}$, Hyung-il Lee ${ }^{b *}$, Jong Mok Park ${ }^{a *}$

${ }^{a}$ Center for Advanced Specialty Chemicals, Korea Research Institute of Chemical Technology (KRICT), Ulsan 44429, Republic of Korea

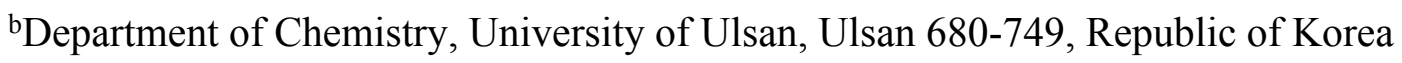

'Korea Research Institute of Standards and Science, Daejeon 305-340, Republic of Korea

dDepartment of Chemistry, and Chemistry Institute for Functional Materials, Pusan

National University, Busan 46241, Republic of Korea. 


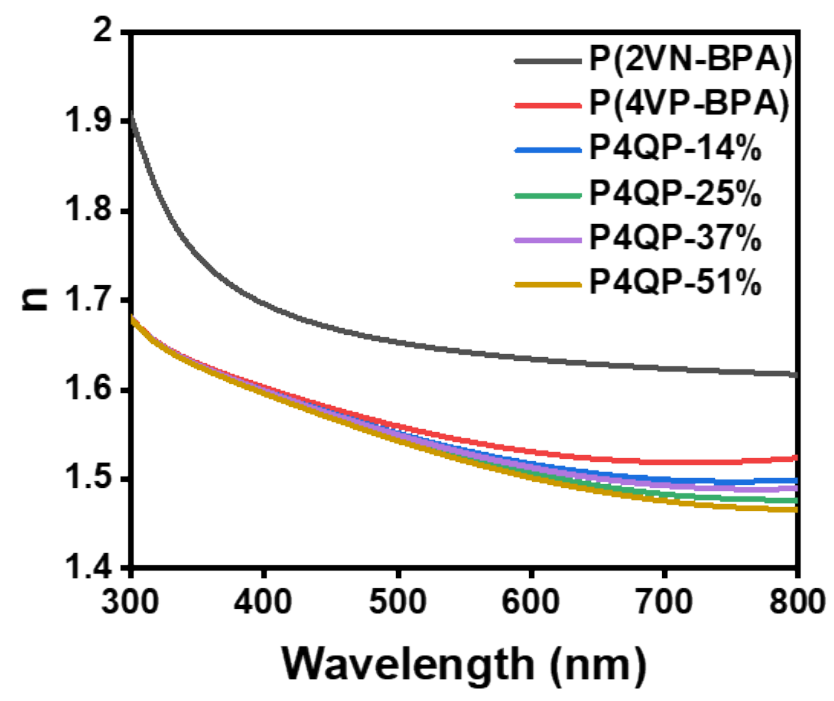

\begin{tabular}{|c|c|}
\hline Polymers & Refractive index \\
\hline P(2VN-BPA $)$ & 1.630 \\
\hline P(4VP-BPA $)$ & 1.530 \\
\hline P4QP-14\% & 1.509 \\
\hline P4QP-25\% & 1.497 \\
\hline P4QP-37\% & 1.504 \\
\hline P4QP-51\% & 1.491 \\
\hline
\end{tabular}

Figure S1. The refractive indices of single layers of each polymer analyzed by ellipsometer.

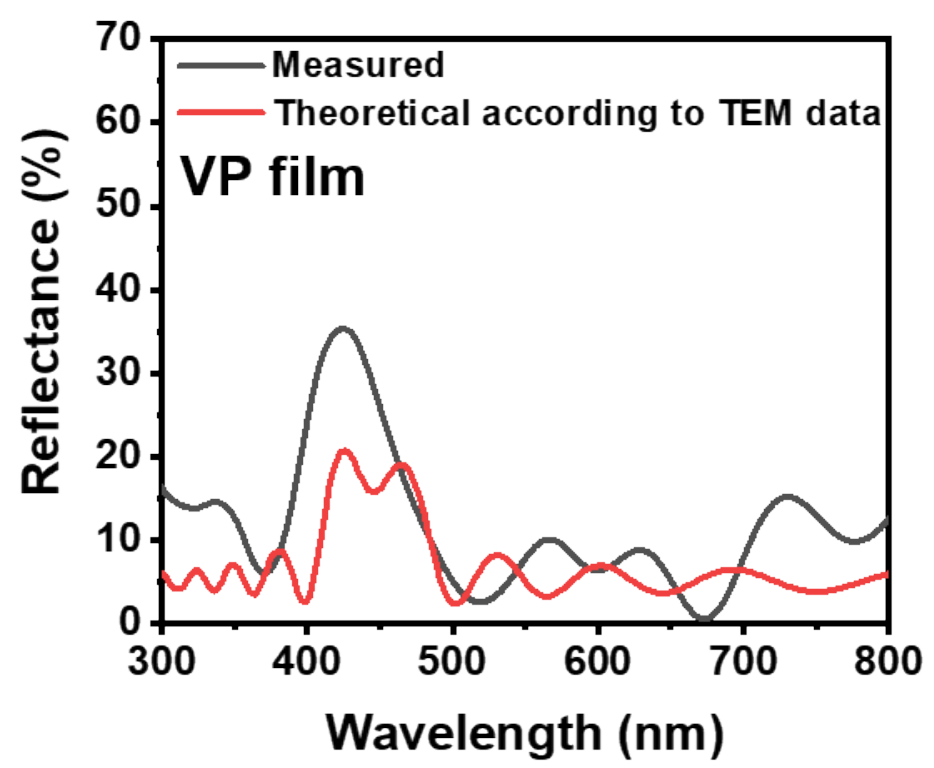

Figure S2. Comparsion of experimental (black) and the simulated (red) reflectance spectra . 
(a)

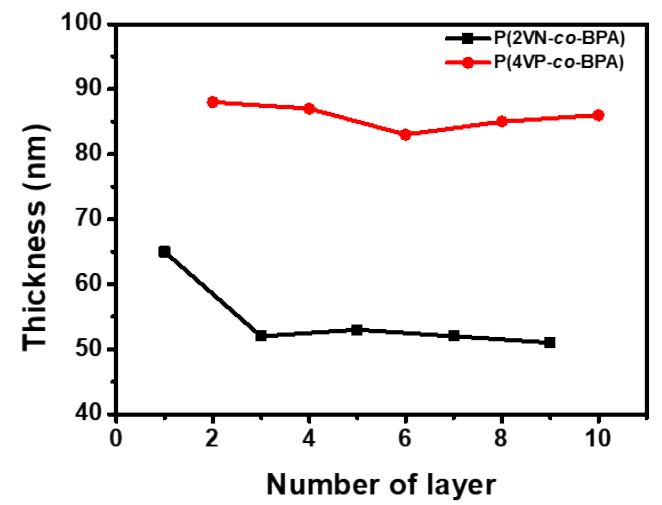

(b)

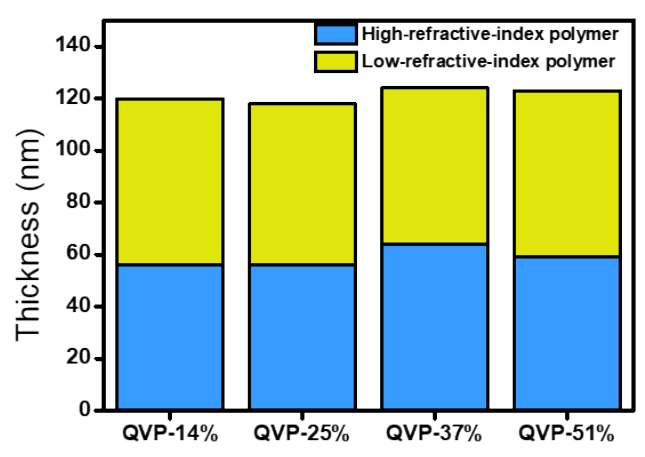

Figure S3. (a) The thickness of each layer of the high- and low-refractive-index polymers in VP (b) the average thickness of each layer of the high- and low-refractive-index polymers in QVP-14\% to QVP-51\%, indicating that the thickness of each layer was consistent and predictable.

(a)

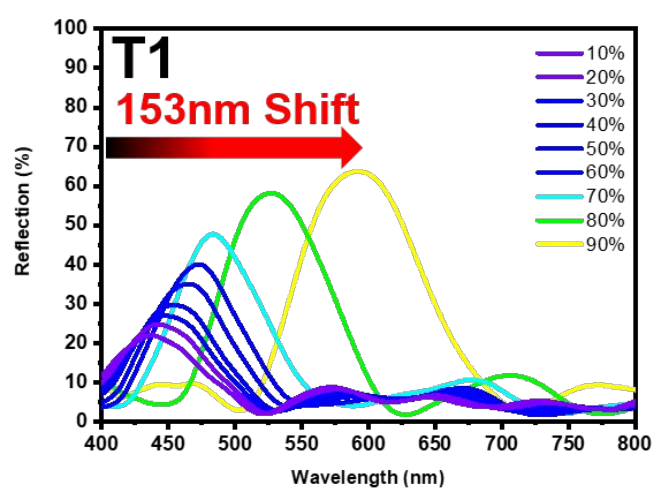

(b)

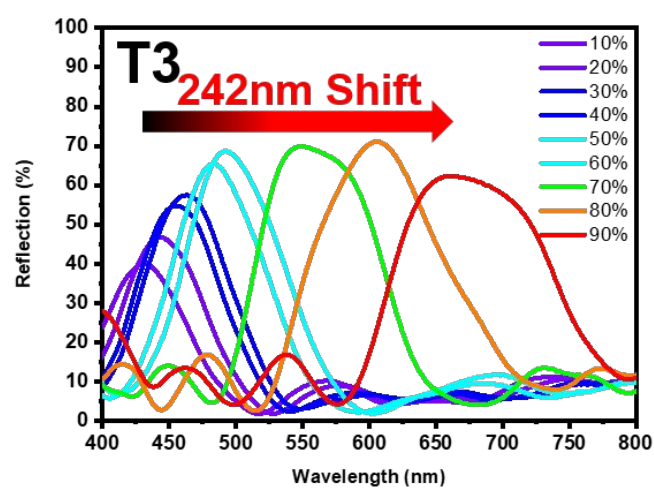

Figure S4. Reflectance spectra of the T1 (a) and T3 (b) samples upon increasing the RH from 10 to $90 \%$, 